\title{
Unidirectional Charge Instability of the $d$-Wave Resonating Valence Bond Superconductor
}

\author{
M. Raczkowski ${ }^{a}$, M. CAPello ${ }^{b}$ And D. PoilblanC ${ }^{b}$ \\ ${ }^{a}$ Marian Smoluchowski Institute of Physics, Jagiellonian University \\ Reymonta 4, PL-30-059 Kraków, Poland \\ ${ }^{b}$ Laboratoire de Physique Théorique UMR5152, CNRS \\ F-31062 Toulouse, France
}

\begin{abstract}
Starting from a uniform $d$-wave superconducting phase we study the energy cost due to imposed unidirectional defects with a vanishing pairing amplitude. Both renormalized mean-field theory and variational Monte Carlo calculations within the $t-J$ model yield that the energies of inhomogeneous and uniform phases are very close to each other. This suggests that small perturbations in the microscopic Hamiltonian might lead to inhomogeneous superconducting phases in real materials as observed in recent scanning tunneling microscopy on $\mathrm{Ca}_{2-x} \mathrm{Na}_{x} \mathrm{CuO}_{2} \mathrm{Cl}_{2}$.
\end{abstract}

PACS numbers: 74.72.-h, 74.20.Mn, 74.81.-g, 75.40.Mg

\section{Introduction}

Recent progress in spectroscopic techniques has provided a wide variety of interesting data concerning electronic states of the high- $T_{\mathrm{c}}$ superconductors. For example, scanning tunneling microscopy (STM) on different cuprate families $\mathrm{Ca}_{2-x} \mathrm{Na}_{x} \mathrm{CuO}_{2} \mathrm{Cl}_{2}$ and $\mathrm{Bi}_{2} \mathrm{Sr}_{2} \mathrm{Dy}_{0.2} \mathrm{Ca}_{0.8} \mathrm{Cu}_{2} \mathrm{O}_{8+\delta}$ has revealed short-range unidirectional charge domains coexisting with inhomogeneous $d$-wave superconductivity [1]. In particular, it has been found that the doped holes primarily enter oxygen sites leading to a bond-centered charge pattern with a period of four lattice spacings. Motivated by this result, we have recently shown [2] that such a charge order might be naturally interpreted in terms of a valence bond crystal [3], i.e., paramagnetic phase with both spatially varying bond charge hopping and short-range antiferromagnetic (AF) correlations. In this case, an inhomogeneous antiphase domain resonating valence bond ( $\pi$ DRVB) phase was obtained by assuming a $\pi$-phase shift in the superconducting (SC) order parameter across domain walls (DWs). While the antiphase solution is particularly intriguing since, in contrast to its inphase counterpart, offers a simple explanation of the suppression of the effective interlayer Josephson coupling observed in some stripe-ordered high- $T_{\mathrm{c}}$ compounds $[4,5]$, both types of the modulation of the SC order parameter are the subject of intense ongoing studies [6-9]. Therefore, in this paper we shall study the energy cost due to imposed defects with a vanishing pairing amplitude (no $\pi$-shift is assumed across the DWs) and compare the resulting charge modulation with the corresponding one found in the $\pi$ DRVB phase.

\section{Model and the approach}

We investigate a $t-J$ model Hamiltonian,

$$
\mathcal{H}=-t \sum_{\langle i j\rangle, \sigma}\left(\tilde{c}_{i \sigma}^{\dagger} \tilde{c}_{j \sigma}+\text { h.c. }\right)+J \sum_{\langle i j\rangle} \boldsymbol{S}_{i} \cdot \boldsymbol{S}_{j},
$$

where $\tilde{c}_{i \sigma}^{\dagger}=\left(1-n_{i,-\sigma}\right) c_{i \sigma}^{\dagger}$ is the Gutzwiller projected electron operator and use a renormalized mean-field theory (RMFT) in which the local constraints of no doubly occupied sites are replaced by statistical Gutzwiller weights $g_{i j}^{t}\left(g_{i j}^{J}\right)$ for hopping (superexchange) processes, respectively [10]. Hence the mean-field Hamiltonian reads,

$$
\begin{aligned}
& H_{\mathrm{MF}}=-t \sum_{\langle i j\rangle, \sigma} g_{i j}^{t}\left(c_{i, \sigma}^{\dagger} c_{j, \sigma}+\text { h.c. }\right)-\mu \sum_{i, \sigma} n_{i, \sigma} \\
& -\frac{3}{4} J \sum_{\langle i j\rangle, \sigma} g_{i j}^{J}\left[\left(\chi_{j i} c_{i, \sigma}^{\dagger} c_{j, \sigma}+\Delta_{j i} c_{i, \sigma}^{\dagger} c_{j,-\sigma}^{\dagger}+\text { h.c. }\right)\right. \\
& \left.-\left|\chi_{i j}\right|^{2}-\left|\Delta_{i j}\right|^{2}\right],
\end{aligned}
$$

with the Bogoliubov-de Gennes self-consistency conditions for the bond- $\chi_{j i}=\left\langle c_{j, \sigma}^{\dagger} c_{i, \sigma}\right\rangle$ and pair-order $\Delta_{j i}=$ $\left\langle c_{j,-\sigma} c_{i, \sigma}\right\rangle=\left\langle c_{i,-\sigma} c_{j, \sigma}\right\rangle$ parameters in the unprojected state. We consider here the so-called modified Gutzwiller factors,

$$
\begin{aligned}
& g_{i j}^{J}=\frac{4\left(1-n_{h i}\right)\left(1-n_{h j}\right)}{\alpha_{i j}+8 n_{h i} n_{h j} \beta_{i j}^{-}(2)+16 \beta_{i j}^{+}(4)}, \\
& g_{i j}^{t}=\sqrt{\frac{4 n_{h i} n_{h j}\left(1-n_{h i}\right)\left(1-n_{h j}\right)}{\alpha_{i j}+8\left(1-n_{h i} n_{h j}\right)\left|\chi_{i j}\right|^{2}+16\left|\chi_{i j}\right|^{4}}},
\end{aligned}
$$

where $\alpha_{i j}=\left(1-n_{h i}^{2}\right)\left(1-n_{h j}^{2}\right), \beta_{i j}^{ \pm}(n)=\left|\Delta_{i j}\right|^{n} \pm\left|\chi_{i j}\right|^{n}$ while $n_{h i}$ are local hole densities. By including the effects of the nearest-neighbor correlations $\chi_{i j}$ and $\Delta_{i j}$ they are 
known to give a better agreement with a more accurate variational Monte Carlo (VMC) technique [2]. Hereafter, we shall assume a typical value $t / J=3$ and fix the doping level $x=1 / 8$. Finally, using unit cell translation symmetry [11], RMFT calculations were carried out on large $256 \times 256$ clusters at a low temperature $\beta J=500$ approaching thermodynamic limit.

\section{Results and discussion}

In Fig. 1 we show the hole profiles as well as the values of the bond- and pair-order parameters across the unit cell found in the $\pi$ DRVB (top) and inphase DRVB (bottom) state. The obtained modulations clearly reflect the competition between the superexchange energy $E_{J}$ and kinetic energy $E_{t}$ of doped holes. However, a detailed charge profile depends on the assumed type of the $\mathrm{SC}$ order parameter. On the one hand, suppression of the pair-order amplitude $\Delta_{i j}$ along the DWs automatically involves a deviation of the bond-order parameter $\chi_{i j}$ from the value found in the areas with finite $\Delta_{i j}$. Remarkably, the deviation is particularly strong in the case of the antiphase SC order parameter. On the other hand, the absence of the $\pi$ shift across the stripe boundary in the DRVB phase allows the system (as confirmed by the VMC method [8]) to avoid a reduction of $\Delta_{i j}$ on the adjacent vertical bonds which remains almost intact. Therefore, the charge redistributes from the hole rich areas with enhanced $\Delta_{i j}$ in the $\pi$ DRVB phase [2], towards DWs with vanishing $\Delta_{i j}$ in the DRVB state (see Fig. 1).
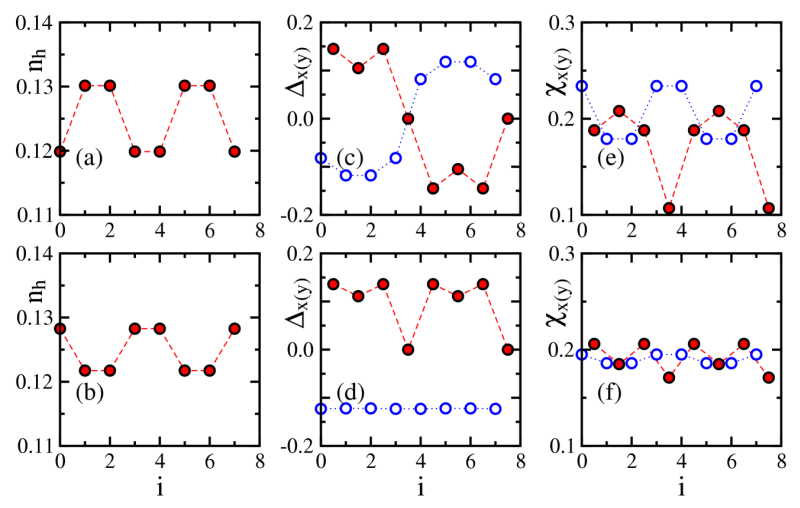

Fig. 1. (a,b) Hole density $n_{h i}$ and variational parameters; (c,d) $\Delta_{i, i+\alpha}$ as well as (e,f) $\chi_{i, i+\alpha}$ found in the $\pi$ DRVB (top) and DRVB (bottom) phase. Solid (open) circles in parts (c)-(f) correspond to the $x(y)$ direction, respectively.

In order to appreciate better the reason of a different charge profile in both phases we show in Fig. $2 \mathrm{a}-\mathrm{d}$ the corresponding short-range AF correlations,

$$
\mathcal{S}_{i}^{\alpha}=-\frac{3}{2} g_{i, i+\alpha}^{J}\left(\left|\chi_{i, i+\alpha}\right|^{2}+\left|\Delta_{i, i+\alpha}\right|^{2}\right),
$$

with $\alpha=\{x, y\}$, as well as bond charge hopping,

$$
\mathcal{T}_{i}^{\alpha}=2 g_{i, i+\alpha}^{t} \operatorname{Re}\left\{\chi_{i, i+\alpha}\right\},
$$

of the SC order parameter (and the concomitant strong suppression of the superexchange energy on the related bonds) enables, in the $\pi$ DRVB phase, a large bond charge hopping along the DWs as in the usual stripe scenario [2]. In fact, it also determines the actual hole profile arranged in the way which minimizes the loss of the superexchange energy at the DWs. This can be easily accomplished by expelling the holes and strengthening locally the corresponding $g_{i j}^{J}$ factors. In contrast, small modulation of $\chi_{i j}$ in the DRVB phase results in a much weaker, with respect to the $\pi$ DRVB one, modulation of both the spin correlations and bond-charge hopping. Consequently, the system does not have to further improve the superexchange energy at the defect lines but it rather tries to regain some kinetic energy released on the broken RVB bonds. This is reached by adjusting the hole profile and attracting the holes to the DWs which enlarges locally renormalization factors $g_{i j}^{t}$. As a result, the DRVB phase has a very good kinetic energy being even slightly better than that of the uniform $d$-wave RVB phase (see Table). Let us point out, however, that even though both the RMFT and VMC methods predict exactly the same hole profiles in the DRVB phase (as well as its remarkably good energy), a discrepancy appears concerning kinetic energy gain at the DWs, strongly enhanced in the VMC method [8]. The difference simply follows from the fact that in the RMFT both the short-range AF correlations and bond-charge hopping are $\propto \chi_{i j}$. Hence its suppression involves a reduction of both the energy contributions unless the system is disposed towards a strong phase separation so that they can be further modified by the Gutzwiller factors [12].
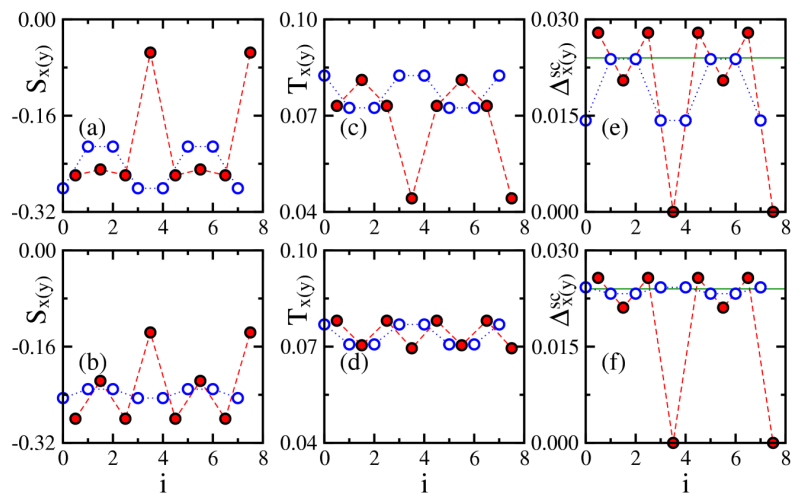

Fig. 2. (a,b) Spin correlation $\mathcal{S}_{i}^{\alpha}$, (c,d) bond charge $\mathcal{T}_{i}^{\alpha}$, and (e,f) SC order parameter $\Delta_{i \alpha}^{\mathrm{SC}}$ found in the $\pi$ DRVB (top) and DRVB (bottom) phase. Solid (open) circles correspond to the $x(y)$ direction, respectively; solid line in parts (e),(f) depicts the SC order parameter in the uniform $d$-wave RVB phase.

Finally, in order to discuss the SC properties of our inhomogeneous phases we plot in Fig. 2e,f the modulus of SC order parameter,

$$
\Delta_{i \alpha}^{\mathrm{SC}}=g_{i, i+\alpha}^{t}\left|\Delta_{i, i+\alpha}\right|,
$$


TABLE

RMFT kinetic energy $E_{t}$, magnetic energy $E_{J}$, and free energy $F$ as well as VMC energy $E_{\mathrm{VMC}}$ of the locally stable phases: $\pi$ DRVB, DRVB, and $d$-wave RVB one at $x=1 / 8$.

\begin{tabular}{c|c|c|c|c}
\hline \hline Phase & $E_{t} / J$ & $E_{J} / J$ & $F / J$ & $E_{\mathrm{VMC}} / J$ \\
\hline$\pi$ DRVB & -0.8719 & -0.4518 & -1.3237 & -1.3359 \\
DRVB & -0.8871 & -0.4662 & -1.3533 & -1.3647 \\
RVB & -0.8863 & -0.4784 & -1.3647 & -1.3669
\end{tabular}

between the $\pi$ DRVB and its inphase counterpart is evident in this figure. Namely, while the SC order parameter deviates, in the regions between defect lines, only slightly in both states from the value found in the uniform $d$-wave RVB phase, the absence of the $\pi$ shift across the stripe boundary in the DRVB phase decouples the horizontal and vertical bonds constituting DWs. Therefore, in contrast to the $\pi$ DRVB phase, the latter retains the value of the $\mathrm{SC}$ order parameter of the uniform state.

\section{Summary and conclusions}

In this paper we have studied two possible modulations of the SC order parameter across the DWs: inphase and antiphase. Remarkably, we have found that the energy of the unidirectional modulated phases (especially of the inphase configuration) approaches the energy of the uniform $d$-wave RVB superconductor. In fact, the energy difference might be further reduced by the tetragonal lattice distortion that often appears in the high- $T_{\mathrm{c}}$ compounds [8]. We conclude therefore that the $d$-wave RVB phase is capable of efficient minimizing the energy cost due to unidirectional defects with broken RVB bonds which in turn might induce the charge modulation similar to that observed in the STM experiments [1].

\section{Acknowledgments}

M.R. acknowledges support from the Foundation for Polish Science (FNP) and from Polish Ministry of Science and Education under project No. N202 068 32/1481. M.C. and D.P. acknowledge the Agence Nationale de la Recherche (France) for support.

\section{References}

[1] Y. Kohsaka, C. Taylor, K. Fujita, A. Schmidt, C. Lupien, T. Hanaguri, M. Azuma, M. Takano, H. Eisaki, H. Takagi, S. Uchida, J.C. Davis, Science 315, 1380 (2007).

[2] M. Raczkowski, M. Capello, D. Poilblanc, R. Frésard, A.M. Oleś, Phys. Rev. B 76, 140505(R) (2007).

[3] S. Sachdev, Nature Phys. 4, 173 (2008).

[4] A. Himeda, T. Kato, M. Ogata, Phys. Rev. Lett. 88, 117001 (2002).

[5] E. Berg, E. Fradkin, E.-A. Kim, S.A. Kivelson, V. Oganesyan, J.M. Tranquada, S.C. Zhang, Phys. Rev. Lett. 99, 127003 (2007).

[6] M. Vojta, O. Rösch, Phys. Rev. B 77, 094504 (2008).

[7] S. Baruch, D. Orgad, Phys. Rev. B 77, 174502 (2008).

[8] M. Capello, M. Raczkowski, D. Poilblanc, Phys. Rev. $B$ 77, 224502 (2008).

[9] M. Vojta, Phys. Rev. B 78, 144508 (2008).

[10] F.C. Zhang, C. Gros, T.M. Rice, H. Shiba, Supercond. Sci. Technol. 1, 36 (1988).

[11] M. Raczkowski, R. Frésard, A.M. Oleś, Phys. Rev. B 73, 174525 (2006); Europhys. Lett. 76, 128 (2006).

[12] M. Raczkowski, D. Poilblanc, R. Frésard, A.M. Oleś, Phys. Rev. B 75, 094505 (2007). 